\title{
Design and Implementation of Cost-effective Temperature Control and hotspot detection in Solar Panels using Aerial Vehicles
}

\author{
Arjun A, Surender Rangaraju, Osama Isaac, Kalai Arul S, Gowthama Krishna D
}

\begin{abstract}
The project's goal is to create a temperature control system for solar panels in a solar farm. As the solar panel is constantly exposed to sunlight, the temperature of the panel steadily rises. Above a particular temperature, the solar cell may be destroyed, and hotspots may form in solar panels. During the course of this project, a gadget for detecting temperature variations is created. Thermal imaging cameras are costly, especially those designed to measure hightemperature objects with minimal measurement error. Lowercost thermal imaging sensors would help a wide range of research and industrial applications. This prototype, attached to a drone, will offer a live feed to a web server presenting a blended frame consisting of a thermographic image exhibiting heat radiation and normal photography with visible light. The platform is made up of a Raspberry Pi single-board computer, a thermal camera sensor, and a standard camera module. At startup, the computer automatically executes Python programmes, initialising its sensor components, and processing the collected photos, which are then broadcast to a live stream through the machine's wifi connection. The goal of this research is to investigate the potential of a low-cost thermal imaging technology for detecting damage in solar panels that may be identified by heat signatures. This project involves the development of an airborne vehicle that will serve as a carrier for thermal cameras used to monitor the temperature of solar panels. The acquired temperature is sent into an Arduino, which controls the solenoid valve that activates the water sprinkler to cool the solar panel. To manage the water level in the sprinkler system's tank, a level control system based on an Ultrasonic sensor and Arduino is built. The effectiveness of solar panels is therefore maintained for a period of time by adopting this control mechanism.
\end{abstract}

Index Terms-Raspberry Pi, Solar Panel, Temperature Control, Thermal Camera

\section{I.INTRODUCTION}

Locating problems in solar panels with thermal sensors such as RTDs and thermocouples is time consuming since a solar farm requires thousands of sensors. The purpose of this study is to look at the possibility of a low-cost thermal

Arjun A - Department of Instrumentation and Control Engineering, PSG College of Technology - India.

Surender Rangaraju - Department of Business and Accountancy Lincoln University College - Malaysia -SNETEL Technologies.

Osama Isaac - Faculty of Business and Accountancy - Lincoln University college - Malaysia.

Kalai Arul S - Department of Instrumentation and Control Engineering, PSG College of Technology - India.

Gowthama Krishna D - Department of Instrumentation and Control Engineering, PSG College of Technology - India imaging method for diagnosing malfunctioning solar cells, which are distinguishable by their heat profiles. A setup like this will be attached to a drone and will provide imagery in the infrared and visible light spectrums as well as the drone's location data. All images and information will be displayed on a laptop via an internet connection to a local server.

\section{II.SYSTEM DESCRIPTION}

IDE Used:

- Python with Thonny IDE

- Arduino programming using Arduino IDE

\section{REQUIRED LIBRARY PACKAGES}

1. LIBRARY OF ADAFRUIT AMG88xx - It is an open source arduino library designed particularly for thermal sensors in the AMG88xx family. It includes modules for collecting thermal camera output and mapping it to an array in order to generate a thermal picture. Its primary purpose is to provide thermal mapping for the AMG8833 sensor.

2. SCIPY - SciPy is a Python library that is free and open-source. It is used for scientific and technical computing. Optimization, linear algebra, integration, interpolation, special functions, FFT, signal and image processing, ODE solvers, and other activities used in research and engineering are all supported by SciPy modules.

3. MATPLOTLIB - Matplotlib is a Python package that allows you to create static, animated, and interactive visualizations. Matplotlib generates publication-quality figures in a range of hardcopy and interactive formats across several platforms. Matplotlib is a graphical user interface toolkit that may be used in Python scripts, the Python and IPython shells, web application servers, and a variety of graphical user interface toolkits.

4. COLOUR - Colour is an open-source Python package that provides a complete collection of colour science methods and datasets. Full RGB, HSL, 6-digit hex, 3digit hex, and human colour conversion. Choosing $\mathrm{N}$ colour gradients for a smooth and intuitive colour scale creation. may use colours to help you identify items in your application.

5. NUMPY - NumPy vectorization, indexing, and broadcasting ideas are today's de-facto array computing standards because they are fast and flexible. NumPy 
provides a wide range of mathematical functions, random number generators, linear algebra routines, Fourier transformations, and other tools. NumPy works well with distributed, GPU, and sparse array libraries and supports a broad range of hardware and computer systems. NumPy's core is well-optimized C code. Take use of Python's flexibility while benefiting from the speed of generated code.

6. PIL (Python Imaging Library) is a free and opensource extension library for the Python programming language that adds support for reading, processing, and saving a wide range of image file types. It is compatible with Windows, Mac OS X, and Linux.

\section{III.PROPOSED METHODOLOGY}

Thermal sensors, aerial vehicles, level control systems, and sprinkler control systems are the most significant concepts in this project. The ideas associated with these notions will now be explained.

\section{THERMAL CAMERA:}

An infrared camera can detect thermal radiation released by surfaces as a result of their temperature. The thermographic effective range of IR detection is typically between MWIR and LWIR. MWIR radiation measurement devices often require cryogenic refrigeration to perform effectively, making them large, costly, and difficult to operate. As a result, most thermographic sensors use the Long Wave Infrared portion of the spectrum. These sensors may function in ambient temperatures, making them far less expensive to build than those used to detect MWIR radiation. When incoming electromagnetic radiation enters a LWIR thermal camera, it is filtered out by a LWIR bandpass film, which is generally made of Germanium or chalcogenide glass. Only light in the LWIR spectrum is transferred and warms the microbolometer components. Every such element has an electrical resistance that is proportional to the amount of incoming radiation it absorbs. Temperature may be determined by sensing these changing resistances.

\section{Infrared and visible light Radiation:}

The wavelength of visible light occupies a small interval in the electromagnetic spectrum, namely from 0.38 $\mathrm{m}$ to $0.74 \mathrm{~m}$, in comparison to infrared (IR) radiation, which spans a larger interval, namely from $700 \mathrm{~m}$ to $1000 \mathrm{~m}$, and can be further classified into: Near Infrared (NIR), Short Wave Infrared (SWIR), Mid Wave Infrared (MWIR), Long Wave Infrared (LWIR), and Far Infrared (FIR).

\section{Raspberry Pi:}

The Raspberry Pi, Model $3 \mathrm{~B}+$ is a tiny single-board computer that is frequently used for educational and development applications. The operating system is the Linux-based Raspbian distribution, which has been optimized for the Raspberry Pi. This computer acts as the main processing unit and was chosen owing to its processor performance, particularly for managing the quick processing of recorded frames and maintaining the stream to a server. It also offers capabilities like WIFI and ready-to-use third- party modules that are simple to deploy.

\section{Raspberry Pi Camera Module:}

The Raspberry Pi camera module, often known as the Pi-camera, is a camera development kit produced by the Raspberry Pi Foundation. It has an 8 Megapixel Color CMOS sensor and can generate photos with a maximum resolution of 32802464 pixels (aspect ratio 4:3). The picamera is simple to use and supports a variety of picture formats. It comes with a well-documented Python library.

\section{GPIO:}

GPIO, which stands for General Purpose Input/Output, is a type of pin that allows simple access to the Raspberry Pi's system from the outside world. As the name implies, it may function as an output pin, sending out digital logic levels to operate an LED light or an electric motor. Reading digital logic signals from an external source while acting as an input pin. Binary threshold voltages are used in communication. When the output is set to HIGH or 1 , the voltage across the pin is increased by 3.3 volts, whereas LOW or 0 equals zero volts. Regarding the input function, the signals must be greater than or equal to 3.3 volts to register as $\mathrm{HIGH}$ or 1 , and zero to register as LOW.

\section{RGB Colour Model:}

RGB is an abbreviation for red, green, and blue, which are the main colours in the additive RGB colour model. The model's premise is to start with a black backdrop and then add proportions between the main components red, green, and blue (RGB) to get any given colour. A colour is represented using decimal code, with each primary colour assigned a value ranging from 0 to 255 (256 total values), which allows it to fit into an 8 -bit byte since $2^{\wedge} 8=256$. The information is written on the form $(\mathrm{R}, \mathrm{G}, \mathrm{B})$. For example, black represents the entire absence of colour $(0,0,0)$ while white represents the full presence of colour $(255,255,255)$. A reasonable analogy would be white light from the sun, which contains all of the colours in the visible spectral range. Red is just used as an example $(255,0,0)$. In many display displays, these three colour values and their arrangement on the screen define each pixel.

\section{AERIAL VEHICLE:}

A quadcopter, sometimes known as a quad-rotor, is a four-rotor aircraft. Because of the tiny size and low inertia of drones, a very simple flight control system may be used, substantially increasing the feasibility of the small quadrotor in this application.

\section{DESIGN PRINCIPLE:}

Each rotor generates lift and torque near its centre of rotation, as well as drag in the opposing direction of the vehicle's flight. Quadcopters typically feature two rotors that rotate clockwise $(\mathrm{CW})$ and two that spin counterclockwise $(\mathrm{CCW})(\mathrm{CCW})$. Flight control is supplied via the independent change of each rotor's speed, and therefore lift and torque. Pitch and roll are regulated by adjusting the net center of thrust, whereas yaw is adjusted by adjusting the net torque. Quadcopters, unlike traditional helicopters, do not often feature cyclic pitch control, in which the angle of 
the blades alters dynamically as they spin around the rotor hub. Quadcopters (previously known as 'quad-rotors' or simply 'helicopters') were viewed as a possible answer to some of the chronic issues with vertical flight in the early days of aviation. Counter-rotation eliminates torque-induced control difficulties (as well as efficiency issues caused by the tail rotor, which provides no usable lift), and the comparatively small blades are considerably easier to manufacture.

If all four rotors spin at the same angular velocity, with two rotating clockwise and two counterclockwise, the net torque at the yaw axis is zero, eliminating the requirement for a tail rotor as in traditional helicopters. Yaw is caused by an imbalance in the balance of aerodynamic torques.

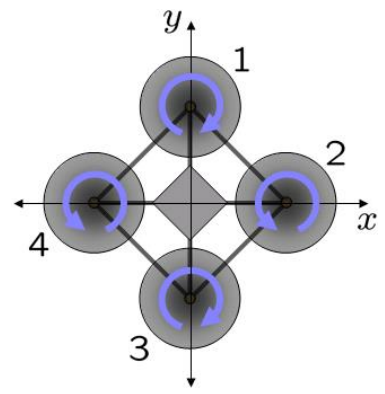

Fig 1: Angular representation of quadcopter

\section{LEVEL AND SPRINKLER CONTROL SYSTEM:}

There are several methods for utilizing a float sensor to determine the water level or probes to detect peak and low levels in the tank. In this project, we used an Ultrasonic sensor and an Arduino to build a level control system. The HC-SR04 serves as a sensing element, and its output is sent to Arduino, which serves as a controller and is used to operate the solenoid valve through a motor driver shield. A LiPo battery is used to power the system (12V). This project's operation is really easy. We utilized an Ultrasonic sensor module that emits sound waves into the water tank and detects sound wave reflection, which is ECHO. First, we must use Arduino to activate the ultrasonic sensor module to broadcast the signal, and then we must wait for ECHO to be received. The time between initiating and receiving ECHO is read by Arduino.

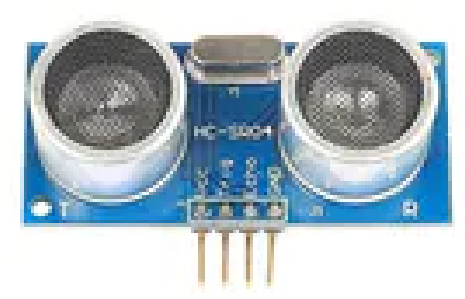

Fig 2: Ultrasonic sensor HC-SRO4

Because we know that the speed of sound is approximately $340 \mathrm{~m} / \mathrm{s}$, we can calculate the distance using the following formula:

Distance $=($ travel time divided by 2$) *$ speed of sound

Where the speed of sound is around 340 meters per second. Using this approach, we can calculate the distance between the sensor and the water's surface. Following that, we must compute the water level.

Thus, the measured level is compared to a threshold level, and if the value is less than the standard value, Arduino sends the signal to the pump to fill the tank, and if the value exceeds a standard upper value, the pump is immediately paused.

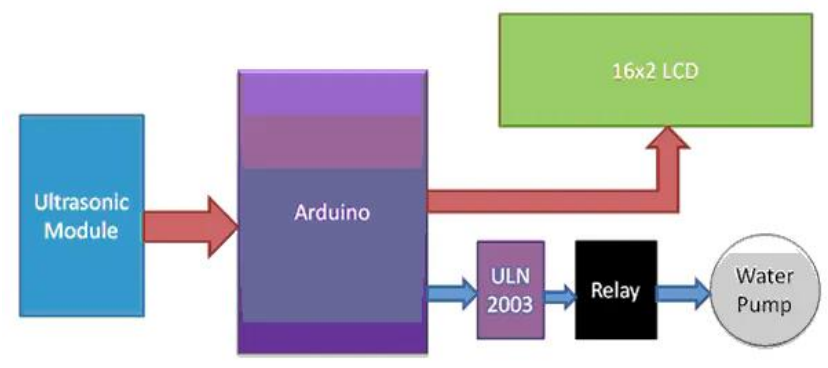

Fig 3: LEVEL CONTROL SYSTEM

\section{IV.IMPLEMENTATION OF PROPOSED SOLUTION}

This chapter describes how to put the recommended solution into action. Python and Arduino programming are used to carry out the implementation. Thonny IDE was chosen as the platform for Python programming.

\section{IMPLEMENTATION:}

1. Temperature Measurement

2. Image Processing

3. Development of quadcopter

4. Interfacing Raspberry Pi with Arduino

5. Water sprinkler control

6. Level control for tank

\section{TEMPERATURE MEASUREMENT}

Materials required are

- Raspberry PI model 3B+

- Panasonic Grid-EYE® Infrared Array Sensors

- Raspberry Pi camera module

- Laptop with VNC viewer

The Raspberry Pi was first introduced by attaching a screen, keyboard, and mouse to it in order to explore the environment in which all software development would take place. Thonny, a Python Integrated Development Environment, was used to write the code. The project's approach was to first connect the camera and install the necessary software. The thermal camera sensor was then connected to the appropriate GIPO pins, and the Adafruit library for using the sensor was installed. With all of the components in place, the next goal was to create a heat picture. Although the Adafruit library included an example script for reading the sensor and translating the temperatures to colours, the moving graphics it produced could not be replicated. As a result, the code was updated to allow image processing, mostly for fusing two frames together. When a final image was obtained, it had to be uploaded to a server.

The initial step was to collect data from the thermal camera. 
- The ADAFRUITS library ADA 8833 was used, which allows for simple sensor reading with the command readPixels(), generating an array containing temperatures in degrees Celsius measured from the sensor's separate elements.

- For the picamera, the function command picamera.capture() generates an image with a specified output file format.

- A reduced resolution of 500X500 pixels was chosen to allow for faster processing and streaming.

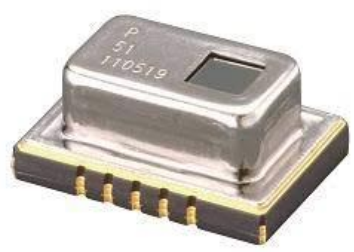

Fig 4: Panasonic AMG8833 Grid

\section{IMAGE PROCESSING:}

- TEMPERATURE PROFILING:

The temperature readings are mapped into a colour gradient spanning from blue to red with all the other colours in between to represent the thermal data in an informative and intuitive manner. When the sensor is read, the lowest temperature is mapped to 0 (blue) and the maximum temperature is mapped to 1023. (red). All of the other temperatures in the period are assigned correlated values.

\section{- INTERPOLATION:}

As previously indicated, the thermal sensor has a rather modest resolution of $8 \mathrm{X} 8$ pixels. Cubic interpolation allowed the resolution to be increased to $32 \mathrm{X} 32$, resulting in a matrix 322/8 $2=16$ times bigger. Interpolation creates new data points by connecting a collection of known points. Depending on the approach utilized, the accuracy may vary. Details happening on the set where a photograph is shot, on the other hand, cannot be reproduced. The original data set is the one obtained following temperature mapping.

\section{- NUMBERING OF IMAGES:}

The RGB colour model converts the values in the $32 \times 32$ matrix spanning from 0 to 1023 into decimal code. The picture may be generated from the decimal code using a function from the SciPy package. When each colour element is counted, the size is $32 \times 32$, but what is presented is an enlarged picture of the original with the form of the individual pixels preserved.

\section{- RESIZING WITH ANTI-ALIASING:}

The next step is to enlarge the $32 \mathrm{X} 32$ picture to a $500 \times 500$ image in order to match the resolution of the camera. When scaling pictures, the Python Image Library (PIL) uses an anti-aliasing filter to "smooth out" the borders between the pixels.

\section{- OVERLAY OF TRANSPARENT IMAGE:}

The camera and heat images are then blended into a single final picture using a prewritten PIL function, merging them together with $50 \%$ transparency on each. It is worth noting that when an image is fused from two sensors separated by a parallel distance, the images do not entirely overlap. Now, the distance is rather small and poses no difficulty as long as items are not directly in front of the sensors. The end output is the picamera image with text and overlaying text indicating the minimum and highest temperature.

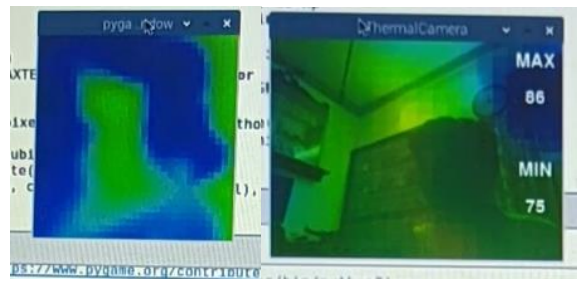

Fig 5 Final Thermal camera output obtained

\section{DEVELOPMENT OF QUADCOPTER:}

Quadcopters have two sets of identical fixed-pitch propellers, two clockwise $(\mathrm{CW})$ and two counter-clockwise (CCW) (CCW). To adjust lift and torque, they employ a change in RPM. The rotation rate of one or more rotor discs is changed to change the torque load and thrust/lift characteristics of the vehicle. The front and back propellers revolve counter-clockwise, while the left and right propellers rotate clockwise. This arrangement of oppositedirection pairs eliminates the requirement for a tail rotor (needed instead of in the standard helicopter structure). Figure 2 depicts the structural model in hovering mode, with all propellers spinning at the same speed. To regulate the hover height of the quadcopter, the PID (ProportionalIntegral-Derivative) control method has been explored and applied in the literature. PID control is a kind of linear control that is commonly used in robotics and automation.

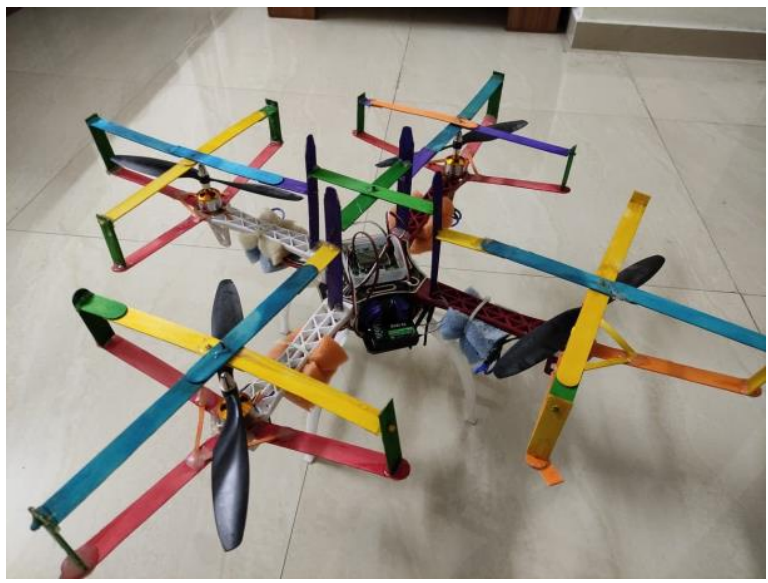

Fig 6: Quadcopter

\section{INTERFACING RASPBERRY PI WITH ARDUINO:}

The solenoid/relay is controlled by nodeMCU pins 13 or D7. The output current of the nodeMCU is insufficient to operate a large load like as a motor, solenoid, or switch, for example. To regulate the output load, a transistor is turned on and off. The transistor also aids in the isolation of the heavy load circuit from the nodeMCU. It protects the 
nodeMCU from the reverse EMF current surge caused by relay coils. The project makes use of an NPN transistor. The $\mathrm{Rb}$ value of a resistor can range from $220 \mathrm{ohm}$ to $1 \mathrm{k} \mathrm{ohm}$. When the power is turned off, a flywheeling diode is attached across the relay coil to protect the circuit from back-induced current. Vs can range between +5 and 24 volts. With this setup, significantly larger loads may be connected to the relay's output.

\section{WATER SPRINKLER CONTROL:}

As a controller, an Arduino UNO is utilized. The Arduino is linked to the Raspberry $\mathrm{Pi}$ in order to get temperature data as input. The signal is then sent to a solenoid valve, which is controlled by an external motor driver (L293D). We used a Solenoid water valve with a nodeMCU ESP8266 Wi-Fi module in this project. The final mechanical gadget is controlled over the internet in this case. NodeMCU is providing a web page as a server. A client device, such as a desktop or a mobile device, is in use. To function effectively, both the server and the client must be connected to the same network (Wi-Fi device).

\section{LEVEL CONTROL OF TANK:}

The primary goal of this system is to measure the level of the water tank and transmit a control signal to switch on the pump when the tank level is low and off when the tank is full. Relays are used to electrically separate two circuits and magnetically link them. When two circuits are entirely isolated, they can be used to switch from one to the other. The relays are made up of an input and an output portion. When a little voltage from an electrical circuit is delivered to the input portion, a coil creates a magnetic field. The ultrasonic sensor module's "trigger" and "echo" pins are directly linked to Arduino, as indicated in the block diagram. The relay is also linked to an Arduino pin for turning on and off the water motor pump.

\section{V.RESULTS AND OBSERVATION}

As a result, the temperature measuring system, aerial vehicle, level, and sprinkler system are developed and built. As we can see, the temperature of the solar panel is kept below the threshold temperature, and therefore its efficiency is kept at the appropriate level. As water is sprinkled over the panel above a certain temperature, we can see that the surface temperature of the panel lowers. Because solar panel efficiency is inversely related to temperature, the efficiency of the solar panel progressively improves.

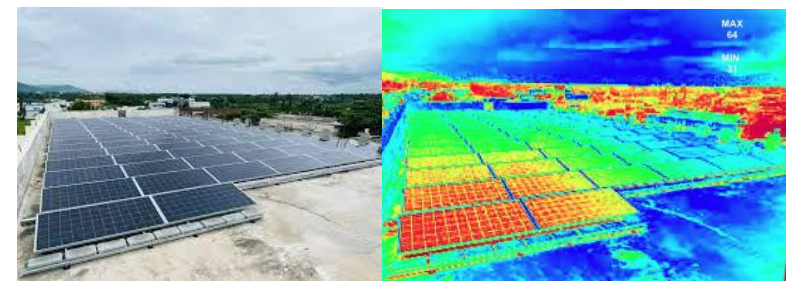

Fig 7: Final thermal imagery of the solar panel

\section{VI.CONCLUSION}

It is apparent that by adopting this management system, the solar panel's efficiency can be maintained at the appropriate level. This method can avoid the formation of hotspots and the deterioration of solar cells. While Panasonic's AMG8833 thermal camera has a suitable field of view, its resolution is not as good as high-resolution thermography, which is required for long-range thermal detection. Panasonic's sensor becomes more appealing when the price is considered.

\section{VII.FUTURE WORK}

- Drone automation can be introduced, reducing human errors at risk.

- The FPS of the thermal camera may be raised, and therefore the system's accuracy can be improved.

\section{REFERENCES}

1. "Introduction to non-contact sensors", Ljubisa Stankovic, Milos Dakovic, Ervin Sejdic, 01 December 2018. [online].

2. Available : https://link.springer.com/chapter/10.1007/978-3-03003574-

7_1\#: :text=Graph\%20signal\%20processing $\% 20$ deals $\% 20$ with,of $\% 2$ 0graphs\%20is\%20discussed $\% 20$ next

3. "Thermal sensors: From Theory to Applications", Yuichi Tanaka, Yonina C. Eldar, Antonio Ortega, Gene Cheung, November 2020. [online].

4. Available: https://signalprocessingsociety.org/publicationsresources/ieee-signal-processing-magazine/thermal-sensorsapplications

5. A. Sandryhaila and J. M. F. Moura, "Implementation of unmanned vehicles," IEEE Trans.automation engineering., vol. 61, no. 7, pp. 1644-1656, Apr. 2013.

6. D. I. Shuman, S. K. Narang, P. Frossard, A. Ortega, and P. Vandergheynst, "The emerging field of signal processing on graphs: Extending high-dimensional data analysis to networks and other irregular domains," IEEE Signal Process. Mag., vol. 30, pp. 83-98, May 2013.

7. A. Sandryhaila and J. M. F. Moura, "Design to implementation of level control system in boiler tank" IEEE Control systems Magazine, vol. 31, no. 5, pp. 80-90, 2014. 